\title{
Electroluminescence from porous silicon due to electron injection from solution
}

\author{
E. S. Kooij, R. W. Despo, and J. J. Kelly \\ Department of Condensed Matter, Debye Institute, Utrecht University, P.O. Box 80 000, 3508 TA Utrecht, \\ The Netherlands
}

(Received 15 December 1994; accepted for publication 20 February 1995)

\begin{abstract}
We report on the electroluminescence from $p$-type porous silicon due to minority carrier injection from an electrolyte solution. The $\mathrm{MV}^{+}$radical cation formed in the reduction of divalent methylviologen is able to inject electrons into the conduction band of crystalline and porous silicon. The electrochemistry of this redox process at silicon electrodes is briefly described, and electroluminescence due to recombination of the injected electrons with holes from the substrate is described. The results are discussed in terms of a semiconductor model. (C) 1995 American Institute of Physics.
\end{abstract}

One of the main reasons for the extensive research on porous silicon is the prospect of integrating optical functions into all-silicon devices. To make a device capable of showing electroluminescence (EL), electrons and holes have to be injected into the porous silicon structure. Radiative recombination of these carriers will give rise to light emission. The first luminescent solid state devices based on this material ${ }^{1-3}$ consisted of a porous layer on a crystalline substrate. A semitransparent Au or transparent conducting oxide electrode was deposited on top of the porous layer. These devices were reported to have very low quantum efficiencies $\left(<10^{-6}\right)$ and require high operating voltages. More recently, different designs $^{4,5}$ and better contact materials ${ }^{6-8}$ have led to some improvement in the electrical and luminescent properties. The reasons for the low EL efficiency are not understood, but it has been suggested that the ease with which carriers are injected into luminescent states and the transport mechanisms in the porous silicon play an important role. ${ }^{9}$

Much higher efficiencies were obtained using a liquid electrolyte junction which contacts the whole porous structure. ${ }^{10-12}$ The mechanism of the strong visible emission from $n$-type porous silicon is thought to be similar to that for conventional semiconductors. Electron capture from the valence band, i.e., hole injection, is achieved by a strong oxidizing agent (electron acceptor) in the solution. At appropriate bias the injected holes recombine with electrons from the conduction band. If this recombination is radiative, EL characteristic of the semiconductor can be observed. ${ }^{13}$

A similar mechanism should hold for $p$-type semiconductors, only now electron injection into the conduction band is required. This is possible if the occupied energy levels of the redox couple in the solution overlap with the conduction band of the semiconductor. Thus, a strong reducing agent (electron donor) has to be used. To our knowledge there have been no reports of EL from $p$-type porous silicon due to minority carrier injection by a reducing agent in solution. ${ }^{14}$ Light emission during anodic oxidation of $p$-type porous silicon in indifferent electrolyte has been reported. ${ }^{15}$ This has been attributed to electrons released during oxidation of $\mathrm{Si}-\mathrm{H}$ surface species or of Si-Si bonds. However, this explanation may be suspect as luminescence is also observed under similar conditions at $n$-type porous layers. ${ }^{12,16}$ Meulenkamp et al. ascribe the emission to electrochemically induced chemiluminescence. $^{12}$

From experiments with other semiconductors ${ }^{17}$ we concluded that the $\mathrm{MV}^{+}$radical cation, which is formed in the reduction of the divalent methylviologen ion $\mathrm{MV}^{2+}$, should be able to inject electrons into porous silicon. The reduction of methylviologen (1, $1^{\prime}$-dimethyl-4, $4^{\prime}$-bipyridinium),

$$
\mathrm{MV}^{2+}+\mathrm{e}^{-} \rightarrow \mathrm{MV}^{+} \cdot
$$

is highly reversible and has a redox potential of $-0.69 \mathrm{~V}$ vs SCE. ${ }^{18}$ In this letter the electrochemistry of methylviologen at silicon electrodes is briefly described, and results of EL due to electron injection by $\mathrm{MV}^{+} \cdot$ into $p$-type porous silicon are presented.

Electrodes were cut from $n$-type $(0.7$ to $0.9 \Omega \mathrm{cm})$ and p-type ( 8 to $12 \Omega \mathrm{cm}$ ) single crystal silicon wafers which had the (100) orientation. Porous layers were formed on $p$-type silicon by anodic etching in a two-electrode configuration using a Pt counter electrode. Anodization was performed galvanostatically in the dark for 5 minutes at $35 \mathrm{~mA} \mathrm{~cm}^{-2}$ in a 1:1 mixture of $20 \% \mathrm{HF}$ :ethanol. Before and after each measurement the presence of the luminescent porous layer was checked by a PL measurement at an excitation wavelength of $354 \mathrm{~nm}$. The electrochemical experiments were performed using an EG\&G Princeton Applied Research (PAR) 366A bipotentiostat in a conventional electrochemical cell containing a Au counter electrode and a saturated calomel electrode (SCE) as reference. All potentials are given with respect to $\mathrm{SCE}$. We used the rotating ring-disc electrode (RRDE) configuration. The working electrode was a silicon ring (4.55 $\mathrm{mm}$ and $6.9 \mathrm{~mm}$ inner and outer diameter, respectively) and a Au disc (4.0 mm diameter) was used to generate the active redox species. The EL measurements were performed using a Si photodiode in combination with an Oriel amplifier (Model 70710). Before each measurement high purity Ar gas was bubbled through the solution to remove oxygen.

Figure 1 shows a potentiodynamic measurement using a p-type crystalline silicon/Au RRDE in $2 \mathrm{mM} \mathrm{MVCl}_{2}+0.1$ $\mathrm{M} \mathrm{KCl}$ aqueous solution in the dark. The ring potential was kept at a fixed value $\mathrm{V}_{\text {ring }}=-0.2 \mathrm{~V}$, while the disc potential was scanned from $-0.2 \mathrm{~V}$ to $-0.95 \mathrm{~V}$. An identical $I-V$ curve was found with $n$-type crystalline silicon, also in the dark. 


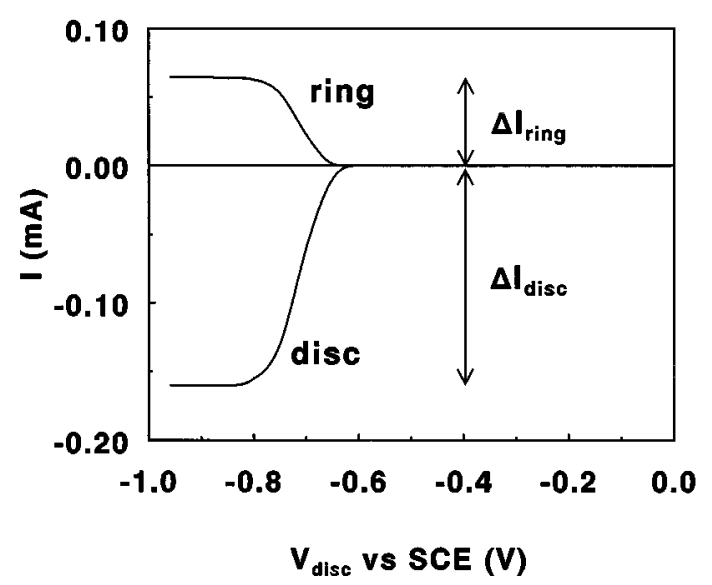

FIG. 1. Potentiodynamic measurement using a $p$-type crystalline silicon/Au RRDE at a fixed ring potential $\left(V_{\text {ring }}=-0.2 \mathrm{~V}\right.$ ) in $2 \mathrm{mM} \mathrm{MVCl}_{2}+0.1 \mathrm{M}$ $\mathrm{KCl}$ aqueous solution. The disc potential was scanned from $-0.2 \mathrm{~V}$ to $-0.95 \mathrm{~V}$ with $10 \mathrm{mV} / \mathrm{sec}$. The rotation rate of the electrode was 1600 revolutions per minute (rpm).

When the potential is scanned towards negative values, the reduction of $\mathrm{MV}^{2+}$ to $\mathrm{MV}^{+}$at the disc starts at $-0.65 \mathrm{~V}$ and reaches a diffusion limited plateau at $-0.8 \mathrm{~V}$. At the same time, an anodic current proportional to the disc current is observed at the ring; this is due to oxidation of the $\mathrm{MV}^{+}$formed at the disc. At $p$-type silicon this current can result either from electron injection into the conduction band or from hole capture from the valence band; the measurements at $n$-type samples in the dark indicate that the current must be due to electron injection. At porous silicon essentially the same $I-V$ characteristics are found, thereby showing that electron injection takes place. The change in the ring current $\Delta I_{\text {ring }}$ as the disc potential is swept from the zero current region to the diffusion limited region (see Fig. 1), depends on the ring potential. This dependence is shown in curve (a) of Fig. 2 for $p$-type porous silicon; $\Delta I_{\text {ring }}$ is nor-

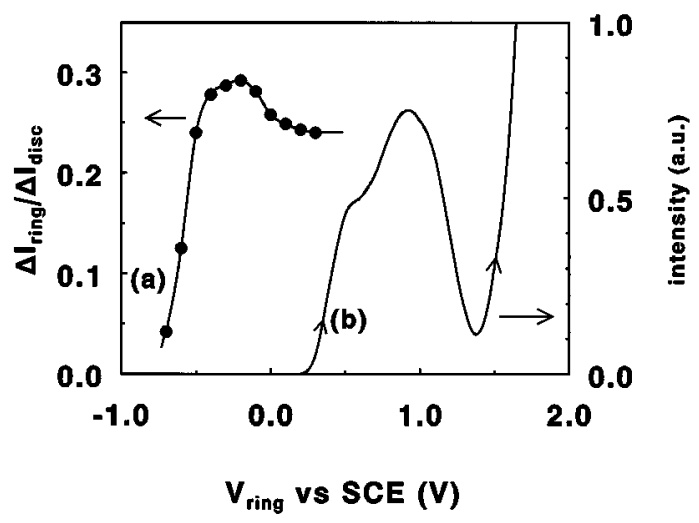

FIG. 2. (a) Potential dependence of the ring current $\Delta I_{\text {ring }}$ of a $p$-type porous silicon electrode, normalized with respect to the change in the disc current $\Delta I_{\text {disc }}$ (conditions as for Fig. 1). (b) Emission-potential curve of a $p$-type porous silicon ring electrode in $0.1 \mathrm{M} \mathrm{MVCl}_{2}+0.1 \mathrm{M} \mathrm{KCl}$ aqueous solution while $\mathrm{MV}^{+}$is generated at the disc at a constant potential. The ring potential was scanned with $10 \mathrm{mV} / \mathrm{sec}$, while the electrode was rotated at 1600 rpm. malized with respect to the change in the disc current, $\Delta I_{\text {disc }}$. Oxidation of $\mathrm{MV}^{+} \cdot$ begins at about $-0.6 \mathrm{~V}$.

To study EL the potential of a $p$-type porous silicon ring was scanned, while $\mathrm{MV}^{+}$was generated at the Au disc at a constant potential. Figure 2, curve (b) shows an emissionpotential curve of the porous silicon ring. In the forward scan, luminescence is measured between $0.2 \mathrm{~V}$ and $1.35 \mathrm{~V}$, only in the presence of $\mathrm{MV}^{+}$. At more positive potentials a strong emission is found, which is related to anodic oxidation of the porous layer. ${ }^{12,15}$ In the return scan no EL is observed at potentials more negative than $1.5 \mathrm{~V}$, which proves that the electrode is indeed oxidized. This is also supported by the fact that the anodic current decreases to zero after polarization at positive potentials. When the potential is scanned to $1.3 \mathrm{~V}$ and back, the emission-potential curves are the same in both scan directions (only slight hysteresis), indicating that the luminescence can indeed be ascribed to electron injection by the reducing agent.

An $\mathrm{MV}^{+}$solution is blue and has a broad absorption band in the visible region of the spectrum with a maximum at $600 \mathrm{~nm} .{ }^{18}$ Unfortunately, this overlaps the emission from porous silicon which is also in the visible with a maximum generally between 600 and $700 \mathrm{~nm} .{ }^{10-12}$ It is therefore difficult to measure a spectrum of the emission.

From Fig. 2 it follows that the EL intensity strongly depends on the applied potential. At negative potentials the surface of the porous silicon is mainly hydrogen-terminated and the porous layer is depleted of holes. As the potential is made more positive, the hole concentration in the porous layer increases. Although electron injection begins at $-0.6 \mathrm{~V}$, EL is observed only at potentials more positive than $0.2 \mathrm{~V}$ (Fig. 2). Obviously electron-hole recombination is nonradiative in this range; a similar effect has been observed with EL of $n$-type porous silicon in solution. ${ }^{10-12}$ At more positive potentials the surface becomes oxidized resulting in a high density of unsaturated surface bonds. This presumably induces a high surface recombination rate, which leads to a decrease of the EL intensity at potentials above $0.9 \mathrm{~V}$. At still more positive potentials the porous layer is completely oxidized, a process which is accompanied by the "anodic" luminescence ${ }^{15}$ mentioned earlier.

The low EL efficiency encountered in solid state devices has been ascribed to a poor contact between the electrode and the porous structure. This structure is inhomogeneous, consisting of "bulk" regions and "quantum-sized" crystallites. It has been argued that the minority carriers are injected preferentially into bulk regions, ${ }^{9}$ which would account for the relatively low intensity of the emitted light. However, using electrolyte contacts there seems to be no problem with carrier injection into luminescent regions provided the energy levels in the solution are favourable. This was shown for $n$-type porous silicon in previous work ${ }^{10-12}$ and has now been found for $p$-type material.

Although the ease with which minority carriers are injected could explain the differences in EL efficiencies in solid state and liquid junction designs, it does not account for the relatively weak luminescence of $p$-type porous silicon between $0.2 \mathrm{~V}$ and $1.35 \mathrm{~V}$ (Fig. 2) compared to the anodic luminescence at more positive potentials and the cathodic 


\section{c-Si Por-Si electrolyte}

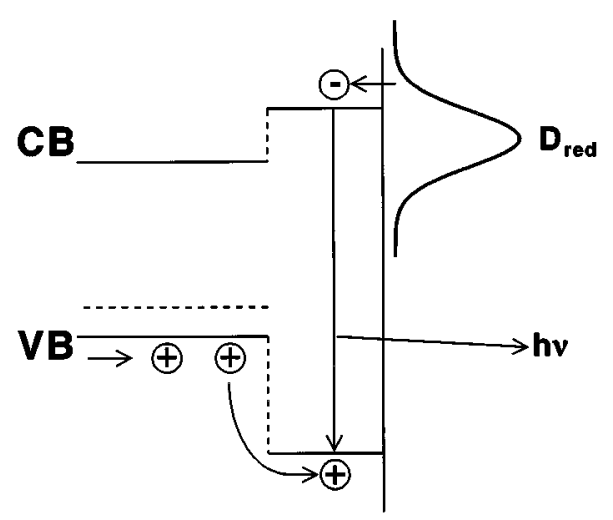

FIG. 3. Schematic representation of the EL mechanism in $p$-type porous silicon as a result of radiative recombination of injected charge carriers. Holes are supplied from the crystalline silicon substrate and electrons are injected by a reducing agent in solution. The distribution of the occupied energy levels $\left(D_{\text {red }}\right)$ is schematically shown.

emission observed at $n$-type electrodes. The band edges of porous silicon do not match those of bulk silicon due to a widening of the band gap. Recently, it has been reported ${ }^{20}$ that the mismatch of the band edges at the silicon/porous silicon junction is more pronounced at the valence band than at the conduction band, $70 \%$ and $30 \%$ of the total mismatch, respectively (Fig. 3). This implies that the barrier for transfer of conduction band electrons from the substrate to the porous layer in $n$-type samples is considerably lower than that for valence band holes in $p$-type samples. Therefore, it seems likely that a higher quantum efficiency can be expected for $n$-type porous silicon. This also seems to be the case for solid state devices, ${ }^{3}$ where the EL properties of $n$-type samples were better than those of $p$ type.

In this work we have demonstrated EL from $p$-type porous silicon due to recombination of holes with electrons injected from a reducing agent in solution. In contrast to solid state devices light emission occurs at a very low cell voltage, as has previously been shown for $n$-type porous silicon in solution.

The authors would like to thank R. Waagenaar and G.H. Schoenmakers for helpful discussions. The work described here was supported by the Netherlands Foundation for Chemical Research (SON) with financial aid from the Netherlands Organization for Scientific Research (NWO).

${ }^{1}$ N. Koshida and H. Koyama, Appl. Phys. Lett. 60, 347 (1992).

${ }^{2}$ F. Namavar, H. P. Maruska, and N. M. Kalkhoran, Appl. Phys. Lett. 60, 2514 (1992).

${ }^{3}$ F. Kozlowski and W. Lang, J. Appl. Phys. 72, 5401 (1992).

${ }^{4}$ Z. Chen, G. Bosman, and R. Ochoa, Appl. Phys. Lett. 62, 708 (1993).

${ }^{5}$ P. Steiner F. Kozlowski, and W. Lang, Appl. Phys. Lett. 62, 2700 (1993).

${ }^{6}$ T. Futagi, T. Matsumoto, M. Katsuno, Y. Ohta, H. Mimura, and K. Kitamura, Appl. Phys. Lett. 63, 1209 (1993).

${ }^{7}$ N. Koshida, H. Koyama, Y. Yamamoto, and G. J. Collins, Appl. Phys. Lett. 63, 2655 (1993).

${ }^{8}$ K. Li, D. C. Diaz, Y. He, J. C. Campbell, and C. Tsai, Appl. Phys. Lett. 64, 2394 (1994).

${ }^{9}$ N. J. Pulsford, G. L. J. A. Rikken, Y. A. R. R. Kessener, E. J. Lous, and A. H. J. Venhuizen, J. Appl. Phys. 75, 636 (1994).

${ }^{10}$ P. M. M. C. Bressers, J. W. J. Knapen, E. A. Meulenkamp, and J. J. Kelly, Appl. Phys. Lett. 61, 108 (1992).

${ }^{11}$ L. T. Canham, W. Y. Leong, M. I. J. Beale, T. I. Cox, and L. Taylor, Appl. Phys. Lett. 61, 2563 (1992).

${ }^{12}$ E. A. Meulenkamp, P. M. M. C. Bressers, and J. J. Kelly, Appl. Surf. Sci. 64, 283 (1993).

${ }^{13}$ E. A. Meulenkamp, J. J. Kelly, and G. Blasse, J. Phys. Chem. 96, 1819 (1992).

${ }^{14}$ E. A. Meulenkamp, T. J. Cleij, and J. J. Kelly, J. Electrochem. Soc. 141, 1157 (1994).

${ }^{15}$ A. Halimaoui, C. Oules, G. Bomchil, A. Bsiesy, F. Gaspard, R. Herino, M. Ligeon, and F. Muller, Appl. Phys. Lett. 59, 304 (1991).

${ }^{16}$ P. C. Searsons, S. M. Prokes, and O. J. Glembocki, J. Electrochem. Soc. 140, 3327 (1993).

${ }^{17}$ G. H. Schoenmakers, R. Waagenaar, and J. J. Kelly (unpublished).

${ }^{18}$ C. L. Bird and A. T. Kuhn, Chem. Soc. Rev. 10, 49 (1981).

${ }^{19}$ M. J. Madou, B. H. Loo, K. W. Frese, and S. R. Morrison, Surf. Sci. 108, 135 (1981).

${ }^{20}$ T. van Buuren, T. Tiedje, J. R. Dahn, and B. M. Way, Appl. Phys. Lett. 63, 2911 (1993) 